Supporting information for

\title{
On-the-fly Symmetrical Quasi-classical Dynamics with Meyer-Miller Mapping Hamiltonian for the Treatment of Nonadiabatic Dynamics at Conical Intersections
}

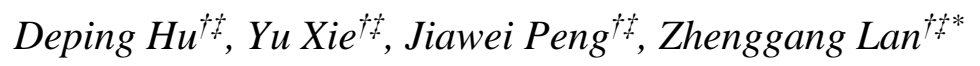

'SCNU Environmental Research Institute, Guangdong Provincial Key Laboratory of Chemical Pollution and Environmental Safety \& MOE Key Laboratory of Environmental Theoretical Chemistry, South China Normal University, Guangzhou 510006, China

${ }^{*}$ School of Environment, South China Normal University, Guangzhou 510006, China

Email: zhenggang.lan@m.scnu.edu.cn; zhenggang.lan@gmail.com 

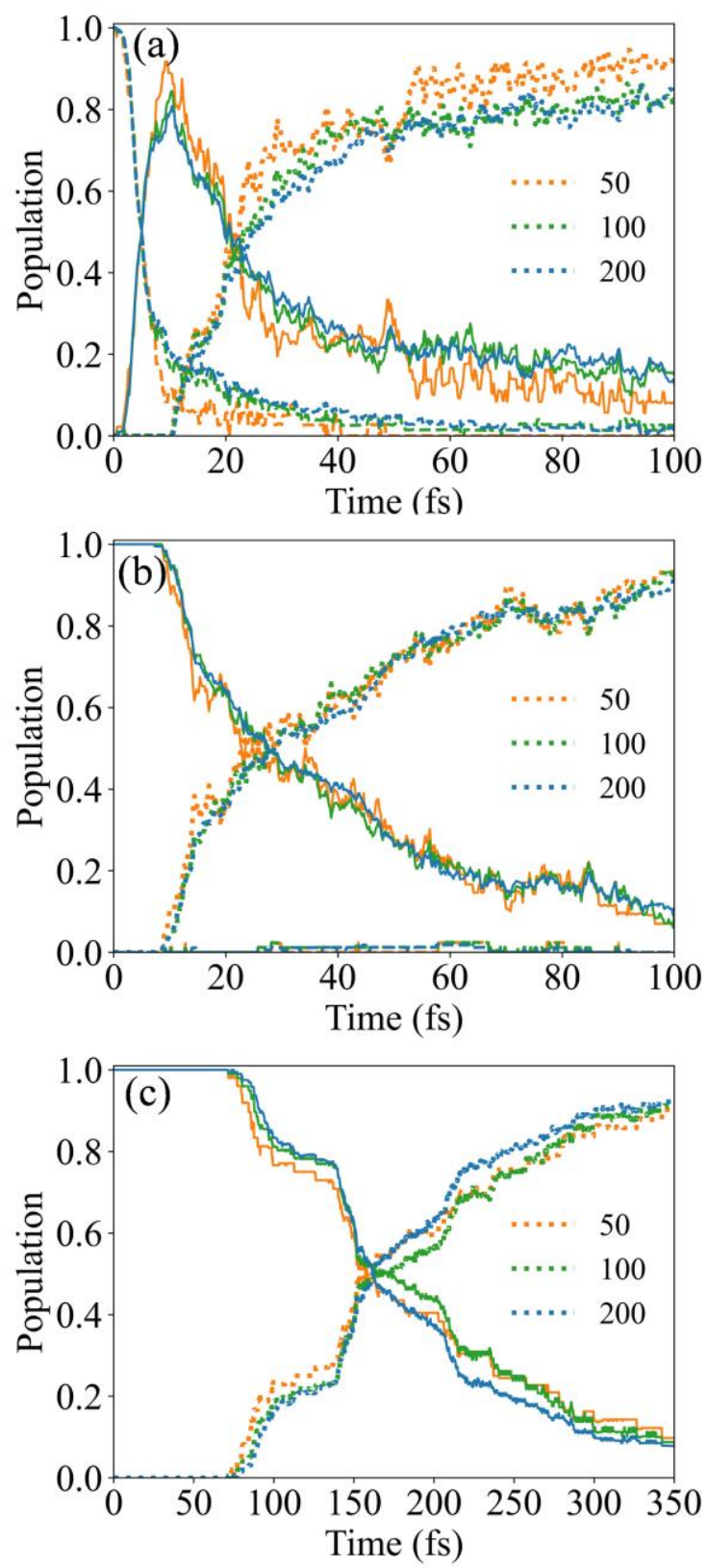

Figure $\mathrm{S} 1$. The time-dependent electronic populations of $\mathrm{S}_{0}$ (dotted line), $\mathrm{S}_{1}$ (solid line) and $\mathrm{S}_{2}$ (dashed line) states of $\gamma$-adjusted SQC/MM dynamics for (a) $\mathrm{CH}_{2} \mathrm{NH}_{2}{ }^{+}$starting from $\mathrm{S}_{2}$, (b) $\mathrm{CH}_{2} \mathrm{NH}_{2}{ }^{+}$starting $\mathrm{S}_{1}$ and (c) azomethane starting from $\mathrm{S}_{1}$. The number of trajectories is given in each subfigure. In the main text, 200 trajectories were used to discuss the results. 
Initial state: $\mathrm{S}_{2}$
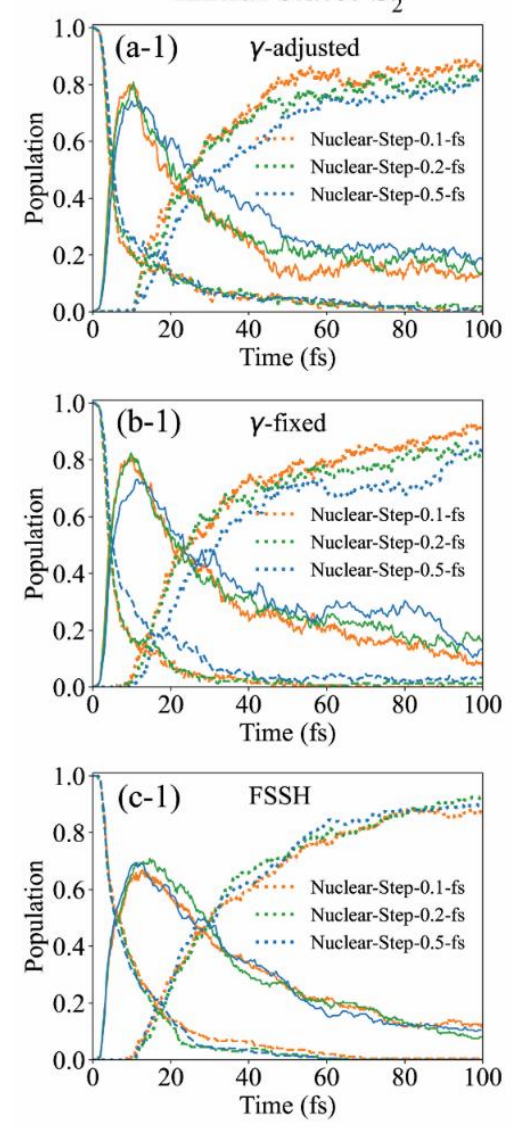

Initial state: $\mathrm{S}_{1}$
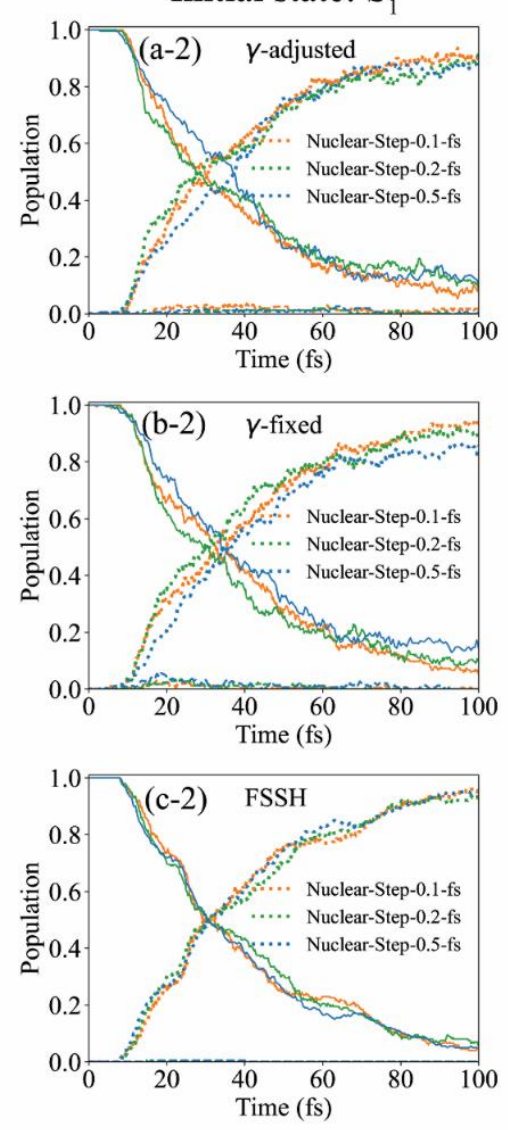

Figure $\mathrm{S} 2$. The time-dependent electronic populations of $\mathrm{S}_{0}$ (dotted line), $\mathrm{S}_{1}$ (solid line) and $\mathrm{S}_{2}$ (dashed line) for (a) $\gamma$-adjusted SQC/MM, (b) $\gamma$-fixed SQC/MM and (c) FSSH dynamics for $\mathrm{CH}_{2} \mathrm{NH}_{2}{ }^{+}$starting from (1) $\mathrm{S}_{2}$ and (2) $\mathrm{S}_{1}$. The numbers in the legends represent the nuclear time steps used in the dynamics. For each step of nuclear motion, 100 steps of electronic motion were performed. 200 trajectories were used for all dynamics.

For SQC/MM dynamics, the time-dependent electronic populations with nuclear time steps of $0.1 \mathrm{fs}$ and $0.2 \mathrm{fs}$ are very close, while a few different results are given with 0.5 fs. For FSSH dynamics, the time-dependent electronic populations change very little from time step of $0.1 \mathrm{fs}$ to $0.5 \mathrm{fs}$. Therefore, we concluded that the nuclear time steps of $0.2 \mathrm{fs}$ and $0.5 \mathrm{fs}$ are enough for SQC/MM and FSSH dynamics, respectively. 

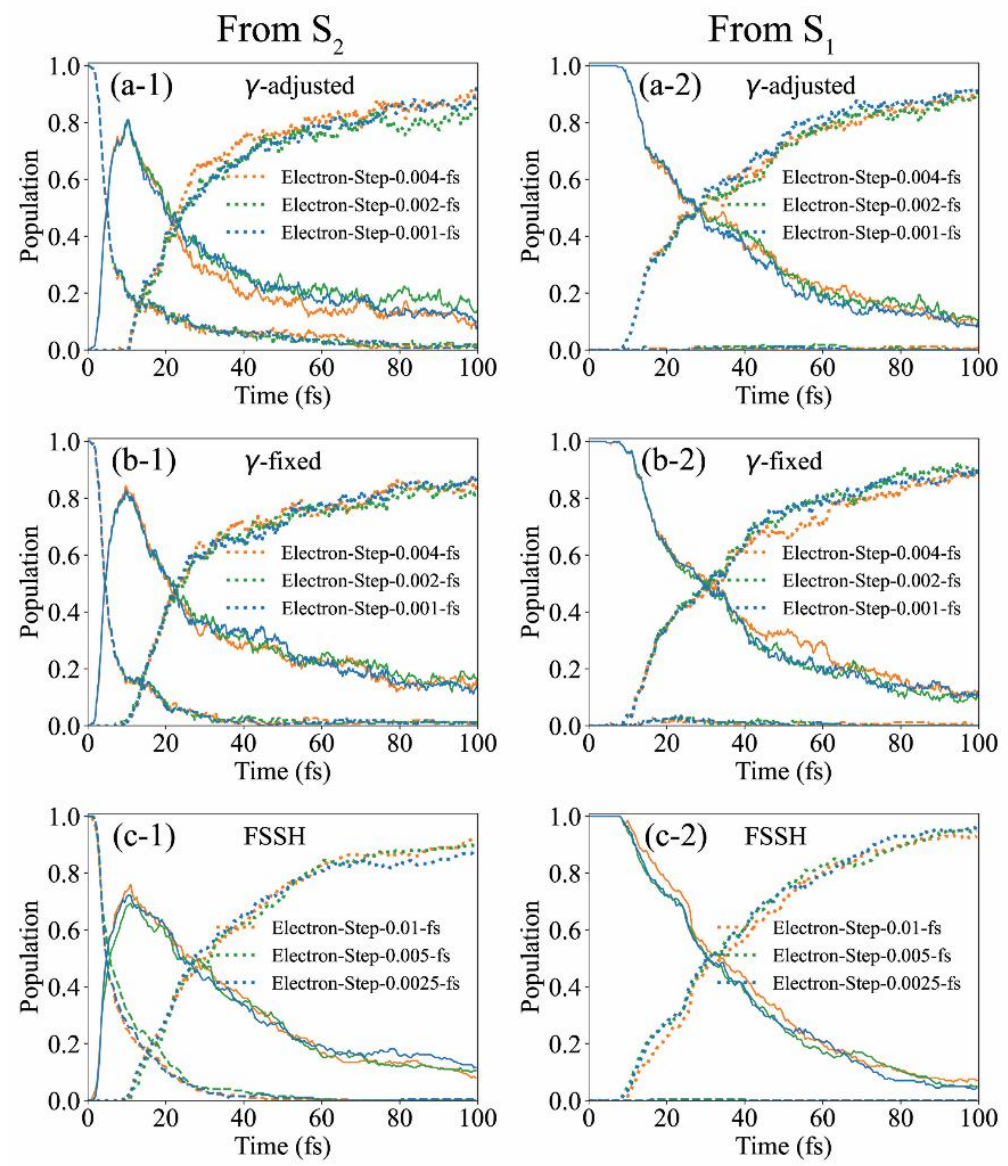

Figure S3. The time-dependent electronic populations of $\mathrm{S}_{0}$ (dotted line), $\mathrm{S}_{1}$ (solid line) and $\mathrm{S}_{2}$ (dashed line) for (a) $\gamma$-adjusted SQC/MM, (b) $\gamma$-fixed SQC/MM and (c) FSSH dynamics for $\mathrm{CH}_{2} \mathrm{NH}_{2}{ }^{+}$starting from (1) $\mathrm{S}_{2}$ and (2) $\mathrm{S}_{1}$. The numbers in the legends represent the electronic time steps used in the dynamics. Nuclear time steps of $0.2 \mathrm{fs}$ and 0.5 fs were used for SQC/MM and FSSH dynamics, respectively. 200 trajectories were used for all dynamics.

For SQC/MM dynamics, the time-dependent electronic populations with electronic time steps of $0.004,0.002$ and 0.001 fs are all very close. For FSSH dynamics, the time-dependent electronic populations with electronic time steps of 0.005 fs and 0.0025 fs are very close, while a few different results are given with 0.01 fs. Therefore, we concluded that the electronic time steps of 0.004 fs (1/50 of corresponding nuclear time step of $0.2 \mathrm{fs})$ and $0.005 \mathrm{fs}$ (1/100 of corresponding nuclear time step of $0.5 \mathrm{fs}$ ) are enough for SQC/MM and FSSH dynamics, respectively. Since the propagation of the electronic motion is not very time-consuming in the on-the-fly dynamics, we used 1/100 of nuclear time steps to obtain the electronic time step for all dynamics. 

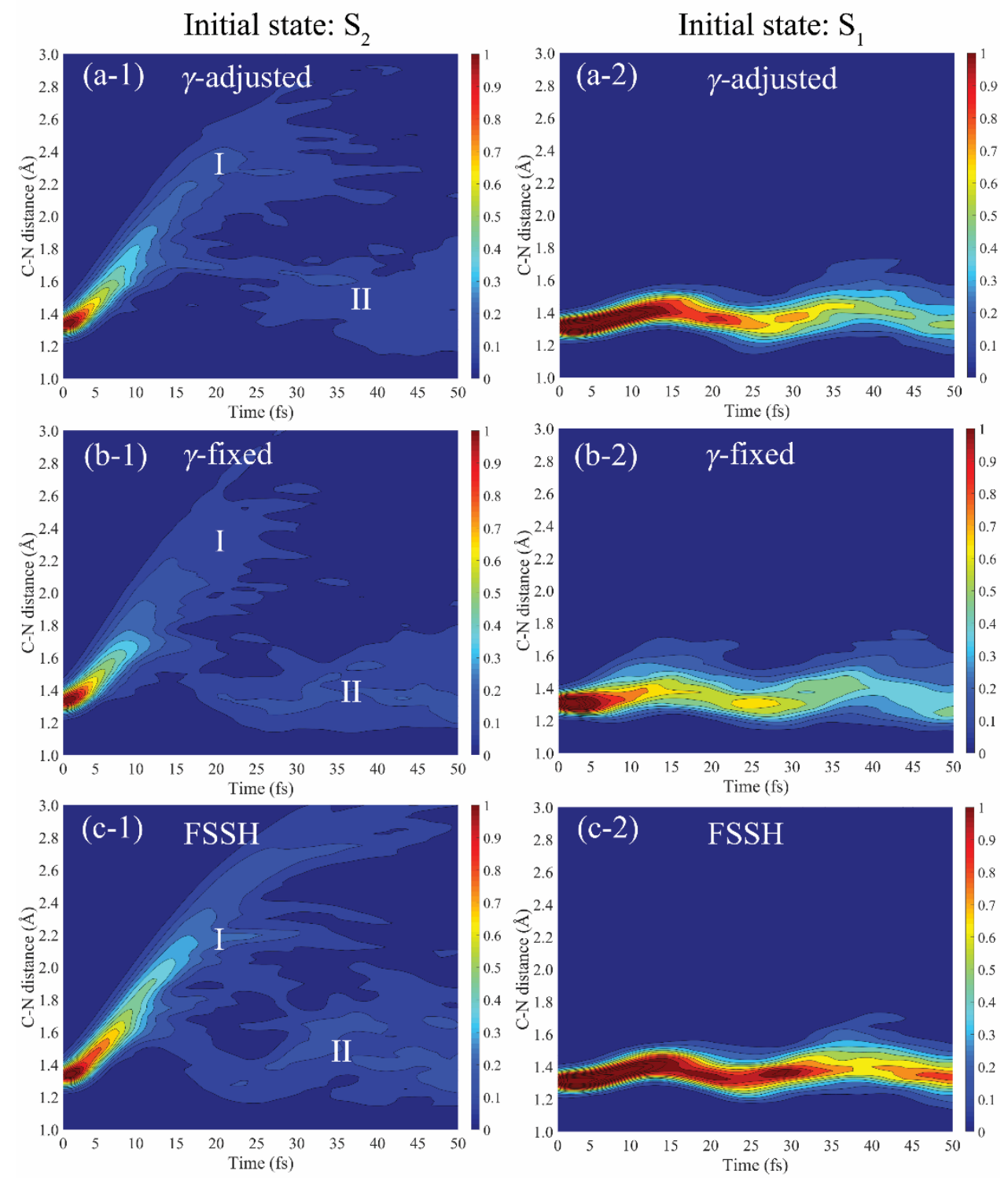

Figure S4. The time-dependent $\mathrm{CN}$ distance distributions of $\mathrm{CH}_{2} \mathrm{NH}_{2}^{+}$in the (a) $\gamma$-adjusted, (b) $\gamma$-fixed SQC/MM and (c) FSSH dynamics. The initial states are (1) $\mathrm{S}_{2}$ and (2) $S_{1}$, respectively. Only the geometries in the windows are included in SQC/MM dynamics. 200 trajectories were used for all dynamics. 

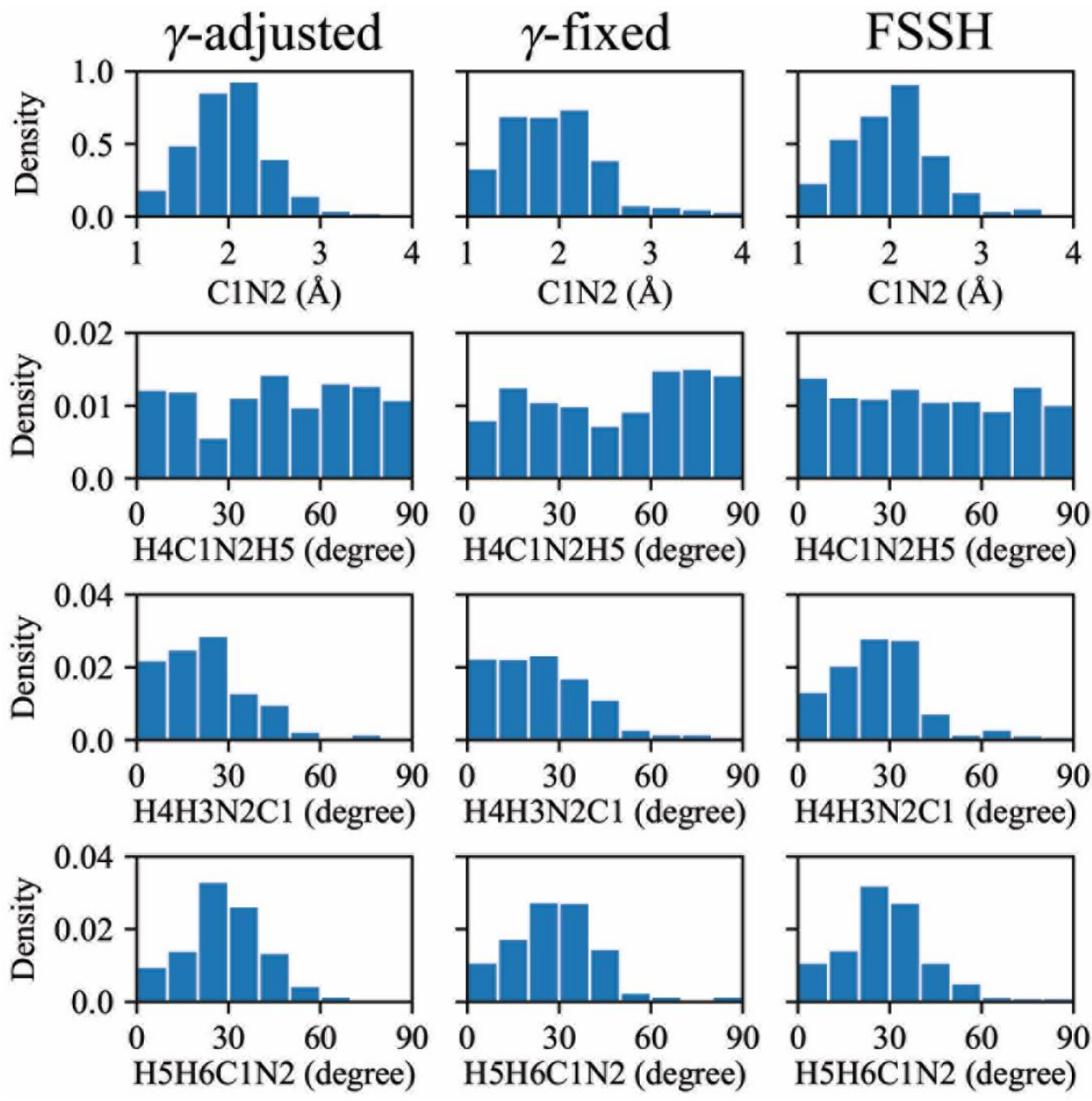

Figure S5. Normalized distributions of key degrees of freedom: bond length C1N2 (CN stretching), dihedral angle $\mathrm{H} 4 \mathrm{C} 1 \mathrm{~N} 2 \mathrm{H} 5$ (CN torsion angle), dihedral angle $\mathrm{H} 4 \mathrm{H} 3 \mathrm{~N} 2 \mathrm{C} 1$ (pyramidalization at $\mathrm{C}$ atom) and dihedral angle $\mathrm{H} 5 \mathrm{H} 6 \mathrm{C} 1 \mathrm{~N} 2$ (pyramidalization at $\mathrm{N}$ atom) of the $\mathrm{CH}_{2} \mathrm{NH}_{2}{ }^{+}$molecule at five $\mathrm{S}_{1} / \mathrm{S}_{0}$ minimum-energy-gap structures in $\gamma$-adjusted SQC/MM, $\gamma$-fixed SQC/MM and FSSH dynamics starting from $\mathrm{S}_{2}$. For all dynamics approaches, we do not consider the trajectory that does not return back $\mathrm{S}_{0}$. 

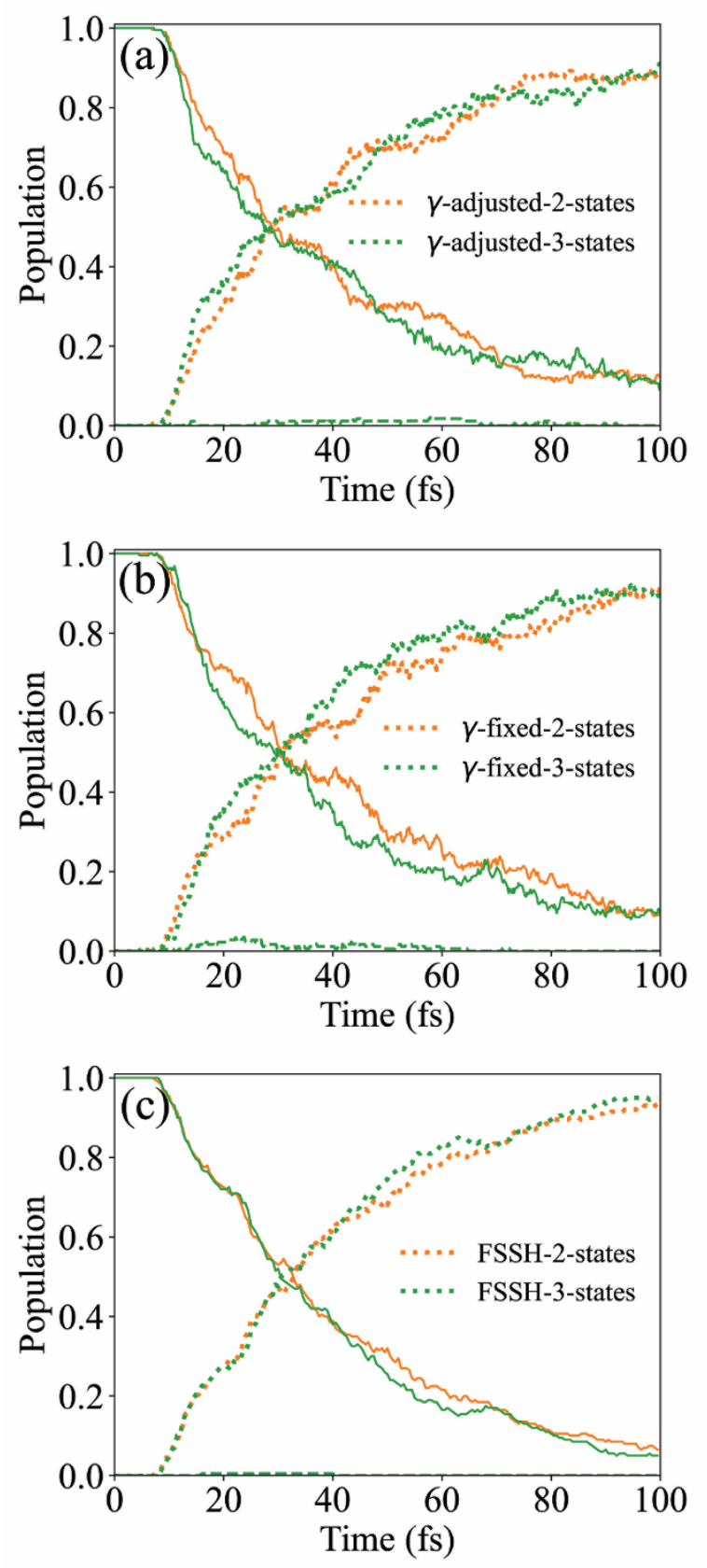

Figure $\mathrm{S} 6$. The time-dependent electronic populations of $\mathrm{S}_{0}$ (dotted line), $\mathrm{S}_{1}$ (solid line) and $\mathrm{S}_{2}$ (dashed line) states of (a) $\gamma$-adjusted SQC/MM, (b) $\gamma$-fixed SQC/MM and (c) FSSH dynamics for $\mathrm{CH}_{2} \mathrm{NH}_{2}{ }^{+}$molecule starting from $\mathrm{S}_{1}$. The number of states involved in the dynamics is given in each subfigure. 200 trajectories were used for all dynamics. 

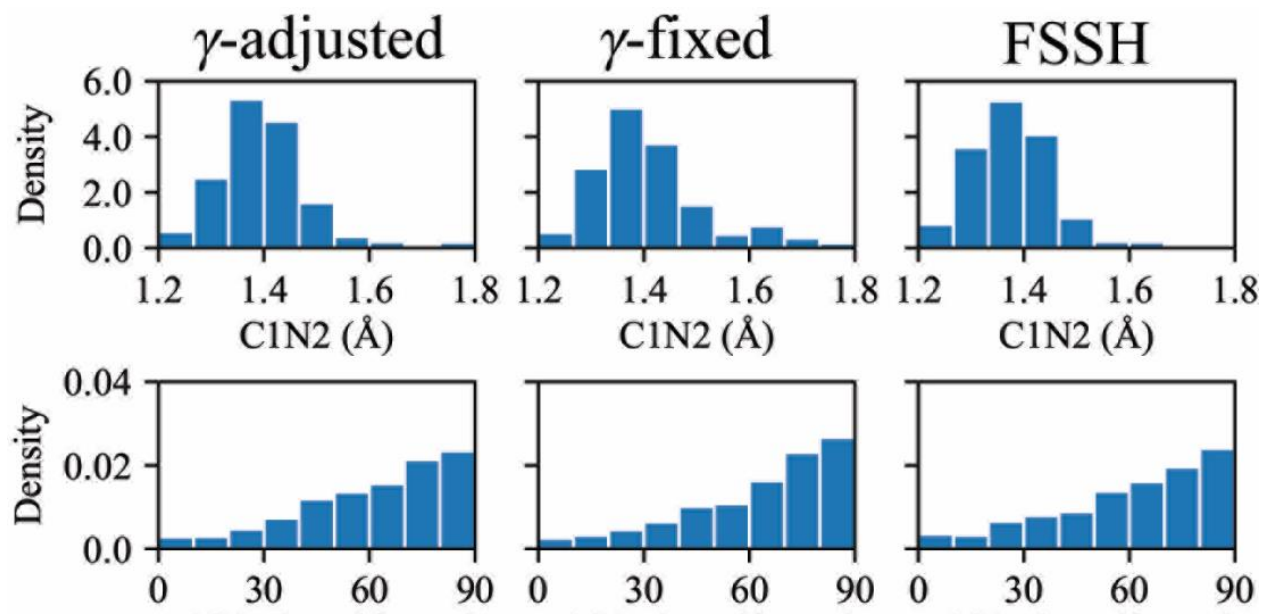

$\mathrm{H} 4 \mathrm{C} 1 \mathrm{~N} 2 \mathrm{H} 5$ (degree)

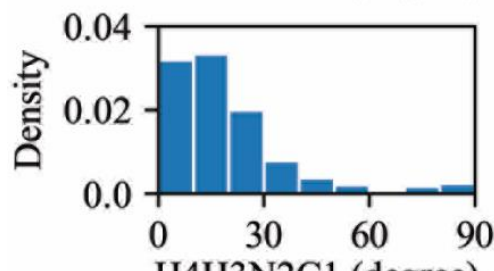

H4C1N2H5 (degree)
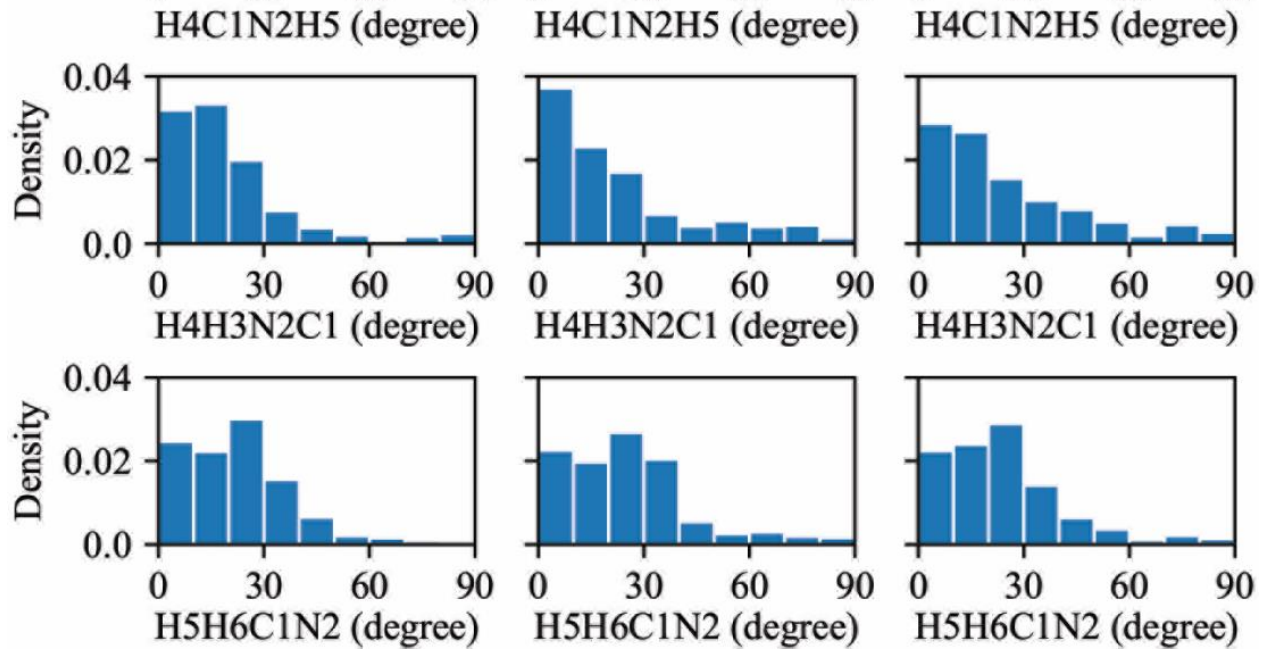

$\mathrm{H} 4 \mathrm{H} 3 \mathrm{~N} 2 \mathrm{C} 1$ (degree)
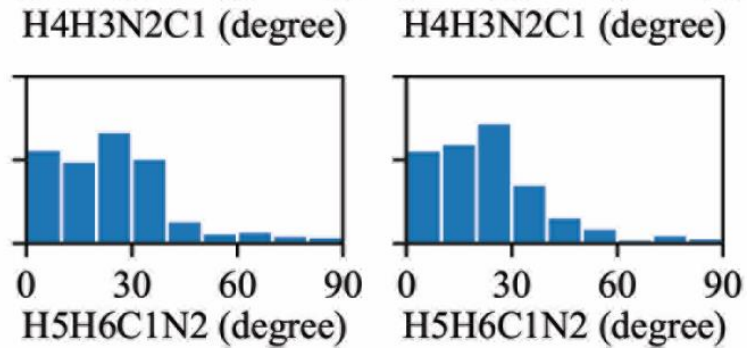

Figure S7. Normalized distributions of key degrees of freedom: bond length C1N2 (CN stretching), dihedral angle $\mathrm{H} 4 \mathrm{C} 1 \mathrm{~N} 2 \mathrm{H} 5$ (CN torsion angle), dihedral angle $\mathrm{H} 4 \mathrm{H} 3 \mathrm{~N} 2 \mathrm{C} 1$ (pyramidalization at $\mathrm{C}$ atom) and dihedral angle $\mathrm{H} 5 \mathrm{H} 6 \mathrm{C} 1 \mathrm{~N} 2$ (pyramidalization at $\mathrm{N}$ atom) of the $\mathrm{CH}_{2} \mathrm{NH}_{2}{ }^{+}$molecule at five $\mathrm{S}_{1} / \mathrm{S}_{0}$ minimum-energy-gap structures in $\gamma$-adjusted SQC/MM, $\gamma$-fixed SQC/MM and FSSH dynamics starting from $\mathrm{S}_{1}$. For all dynamics approaches, we do not consider the trajectory that does not return back to $S_{0}$. 